REGARDS

SUR L'ECONOMIE ALLEMANDE

BULLETIN ECONOMIQUE DU CRAC

\section{Regards sur l'économie allemande}

Bulletin économique du CIRAC

$87 \mid 2008$

Varia

\title{
Pauvreté ? Non, mais la classe moyenne fond
}

Isabelle Bourgeois

\section{OpenEdition}

Journals

Édition électronique

URL : http://journals.openedition.org/rea/983

DOI : $10.4000 /$ rea. 983

ISBN : 978-2-8218-0870-6

ISSN : 1965-0787

Éditeur

CIRAC

Édition imprimée

Date de publication : 1 juillet 2008

Pagination : 38-39

ISSN : 1156-8992

Référence électronique

Isabelle Bourgeois, «Pauvreté ? Non, mais la classe moyenne fond », Regards sur l'économie allemande [En ligne], 87 | juillet 2008, document 2, mis en ligne le 01 juillet 2010, consulté le 15 septembre 2020. URL : http://journals.openedition.org/rea/983

Ce document a été généré automatiquement le 15 septembre 2020

(C) CIRAC 


\title{
Pauvreté ? Non, mais la classe moyenne fond
}

\author{
Isabelle Bourgeois
}

1 Après des semaines de controverses, le conseil des ministres fédéral a adopté le 25 juin le " $3^{\mathrm{e}}$ Rapport du gouvernement sur la pauvreté et la richesse» (3. Armuts- und Reichtumsbericht der Bundesregierung), mieux connu sous le nom de "rapport sur la pauvreté ». Etabli sur la période 2003/05, il dresse un portrait social de l'Allemagne plutôt favorable en comparaison de la moyenne des pays de l'OCDE, les inégalités de revenus étant fortement lissées outre-Rhin par un système généreux de transferts sociaux et fiscaux. La principale conclusion du rapport, établi sous la houlette du ministère fédéral de l'Emploi, est présentée ainsi par ce dernier : «l'Etat social allemand est efficace» (www.bmas.de).

\section{Risque de pauvreté pour $13 \%$ des Allemands}

2 En effet, grâce à ces transferts, le risque de tomber au-dessous du seuil de pauvreté (soit $60 \%$ du salaire médian : $781 €$ net par mois en Allemagne) est tombé de $26 \%$ à $13 \%$ en 2005 (la moyenne européenne est de $16 \%$ ). Chez les enfants, il a été ramené de $34 \%$ à $12 \%$. Les catégories les plus menacées par la pauvreté sont les chômeurs, les non qualifiés, les familles monoparentales et les immigrés. Ce constat-là n'est pas mis en doute, bien que nombre d'experts regrettent le changement de méthode statistique (EU-SILC) qui interdit toute comparaison avec les rapports précédents.

\section{Pourquoi maintenant des données 2005 ?}

3 La principale critique qu'il s'attire porte sur la période retenue : les années de marasme économique (et de réorganisation du dispositif d'indemnisation des chômeurs, voir REA 68/04) où le nombre de demandeurs d'emploi avait atteint le niveau record de 5,3 millions en février 2005. Soit deux millions de plus qu'en 2008. Si ce décalage est normal en matière de statistiques, l'interprétation à laquelle donnent lieu ces données 
historiques, voire l'instrumentalisation qui en est faite dans le contexte politique actuel n'a rien d'innocent.

\section{Amalgame entre pauvreté et risque de pauvreté}

4 Car le constat de l'efficacité de l'Etat social allemand n'atteint pas l'opinion, qui ne retient, par l'intermédiaire des grands médias que ceci : un Allemand sur 8 vit dans la pauvreté. Il est vrai que, de 2002 à 2005 , le salaire brut moyen a baissé de $4,8 \%$ pour tomber à $23684 €$. Mais on confond dans le débat salaire et revenu, ainsi que le rappelle par exemple Hans-Werner Sinn, patron de l'institut ifo, dans une prise de position en date du 9 juin (www.ifo.de). Et on fait l'amalgame entre pauvreté ( $40 \%$ du salaire médian) et risque de pauvreté ( $60 \%$ ). Le seuil de pauvreté, en Allemagne, était fixé, en 2005 , à $520 €$. Si on prend ce seuil pour référence, environ $4 \%$ seulement de la population allemande est pauvre (un taux qui semble incompressible depuis toujours; Sinn) ; mais elle bénéficie de l'aide sociale, un droit garanti par la Constitution.

\section{Instrumentalisation du rapport pour forcer un SMIC légal}

$5 \quad$ Le risque de pauvreté se trouve aujourd'hui instrumentalisé par le SPD et Die Linke pour promouvoir dans l'opinion d'une part une révision à la hausse de ce RMI allemand qu'est l'Arbeitslosengeld II (« Hartz IV »; voir REA 68/04) dont le montant moyen versé à un célibataire s'élevait en 2005 , tous transferts confondus, à $700 €$ (plus l'assurance maladie ; OCDE) mais dont on ne retient que l'allocation forfaitaire de base : $345 €$. Et, d'autre part, pour mieux justifier l'institution d'un salaire minimum légal dans certaines branches comme les services postaux ou l'intérim (voir REA 85/08 et 86/08), voire sa généralisation. L'argument venant en appui de cette revendication, défendue expressis verbis par le ministère fédéral de l'Emploi (SPD) et qui est l'un des objectifs politiques de la publication de ce rapport, réside dans l'extension du segment des bas salaires (mini-jobs, notamment).

\section{Surenchère et dramatisation pour « rétablir la justice sociale »}

Plusieurs autres études viennent à l'appui de cette stratégie, ainsi une récente analyse de l'Institut für Arbeit und Qualifikation de l'Université de Duisburg-Essen, qui affirme que, avec un taux de $22 \%$ de working poor, l'Allemagne talonne les Etats-Unis ( $25 \%)$. Ce que les chiffres ainsi distillés dans l'espace public ne permettent pas de voir, c'est que le grossissement de la catégorie des faibles revenus, qui culmine en 2005, est l'effet arithmétique du changement de l'articulation entre le régime de l'aide sociale et de celui des transferts aux chômeurs en fin de droits (loi Hartz IV), et qu'il est donc exceptionnel. Rien ne permet d'extrapoler ces données et d'en tirer des conclusions sur la situation actuelle, normalisée. Or c'est ce que font ces études et de nombreuses autres. On comprend dès lors mieux que la confédération syndicale DGB, par exemple, s'insurge contre une réalité sociale hautement injuste, forçant des millions d'Allemands à vivre dans la pauvreté bien qu'ils aient un emploi. «Les chiffres publiés sont sans appel, le 
politique doit faire plus contre la pauvreté et pour la justice sociale", s'insurgeait ainsi Annelie Buntenbach, membre du directoire du DGB (Süddeutsche Zeitung, 19-05). Le monde syndical et associatif réclame d'urgence le retour en arrière sur les réformes Hartz et l'institution d'un SMIC légal..

\section{Situation meilleure en 2008 qu'en 2005 grâce à la reprise}

7 Le ministre fédéral de l'Economie, Michael Glos (CSU), tente de calmer les esprits. « $L a$ réalité sociale de l'année 2008 est totalement différente de celle de 2005 ", explique-t-il dans un communiqué publié le jour de la présentation du «Rapport sur la pauvreté» (www.bmwi.de), «ce qui ne permet pas de voir les progrès en matière sociale réalisés au cours des dernières années». Car, ajoute-t-il, "la reprise économique a profité aux Allemands", rappelant que, depuis $2005,38 \%$ des demandeurs d'emploi ont retrouvé du travail. Et il reste farouchement opposé à toute idée de SMIC légal.

\section{Les revenus effectifs sont en hausse depuis $2000 \ldots$}

De son côté, l'Institut der Deutschen Wirtschaft, proche du patronat, a publié le 22 mai une étude sur l'évolution des revenus effectifs des Allemands entre 2000 et 2007 (iwd 21/08). Elle prend en considération non pas les salaires conventionnels de branche, comme le fait l'office fédéral des Statistiques, mais les rémunérations brutes effectivement versées par les employeurs. Alors que les salaires conventionnels ont crû de $12 \%$ en moyenne sur la période - une hausse immédiatement absorbée par l'augmentation du coût de la vie - les revenus effectivement perçus par les salariés ont connu une hausse bien supérieure. Ainsi, celle-ci a été de $18 \%$ dans l'industrie, de $22 \%$ dans le secteur bancaire et même de $27 \%$ dans le commerce de gros. Ainsi, par exemple, le salaire brut effectif moyen atteignait $3926 €$ par mois dans la production d'équipements informatiques (+27,7\%), $3829 €$ dans le secteur bancaire $(+22,3 \%)$, $3687 €$ dans la construction automobile ( $+20 \%$ ), mais seulement $2707 €$ dans le secteur de la vente automobile $(+11 \%)$, les rémunérations les plus faibles se trouvant dans l'agroalimentaire (2 $333 €,+11,9 \%)$.

\section{... mais la classe moyenne est en train de fondre}

La lecture des disparités salariales plus fortes constatées dans les divers rapports et études ne se fait pas seulement sous l'angle de la hausse du nombre des working poor ou des Allemands menacés de pauvreté. Au centre des débats se trouve en effet leur corollaire : la «fonte» de la classe moyenne. L'institut DIW de Berlin fait par exemple état d'une baisse de 3,8 points de pourcentage de la catégorie des classes moyennes (revenus situés entre $150 \%$ et $70 \%$ du médian), entre 2002 et 2005. Ces données sont scientifiquement établies, et donc fiables. Or dans le contexte actuel, elles servent elles aussi à nourrir les revendications les plus diverses, généralement centrées sur la redistribution pour remettre en marche un ascenseur social considéré comme en panne. Et, plus sérieusement, pour rappeler la nécessité d'une politique de formation et de qualifications plus adéquate. 


\section{Des disparités principalement d'origine socio- démographique}

10 Or si cette «fonte » est indéniable, et si la formation est bel et bien une priorité pour développer la prospérité de l'Allemagne et des Allemands, l'accroissement des disparités a une autre origine que celle qu'on lui prête communément (économie de marché, mondialisation, régime d'indemnisation trop strict, dumping salarial, etc.). C'est ce que démontre une autre étude encore, publiée en juin par l'Institut für Wirtschaft und Gesellschaft (www.iwg-bonn.de). C'est en effet aussi et avant tout l'évolution démographique qui modifie les équilibres: la population qualifiée, de souche allemande, est en recul (de 2,8 millions entre 1996 et 2006), celle d'origine étrangère, souvent peu qualifiée, est en hausse (3,5 millions de plus). Or si une partie de cette souche allemande a bénéficié de l'ascenseur social (1,1 million de couples avec enfants et 1 million de plus de 64 ans), sortant ainsi du périmètre des classes moyennes vers le haut, celui-ci a été rogné vers le bas par une arrivée massive de moins qualifiés ( $83 \%$ de la population issue de l'immigration entre dans la catégorie des faibles revenus) et, du fait de l'éclatement des structures familiales en Allemagne aussi, par la hausse du nombre des familles monoparentales (elles sont aujourd'hui plus de 0,8 million). Or ces dernières constituent la majorité du 1,1 million d'Allemands de souche venus grossir la catégorie des bas salaires.

11 Cette étude apporte un peu plus d'objectivité dans le débat politique sur la "pauvreté » des Allemands, avivé par l'inflation et le recul du pouvoir d'achat. Mais si la montée de "l'injustice sociale» interpelle la sensibilité de l'opinion, le politique hésite entre 'surfer' sur cette tendance à l'éclatement ou rassembler la base de son électorat: le « centre » de la société, cette classe moyenne qui fait la compétitivité allemande. C'est là ce qui a motivé le président Horst Köhler à centrer son discours du 17 juin sur : "Travail, Formation, Intégration ».

INDEX

Mots-clés : pauvreté, pouvoir d'achat, salaire, société, système social 